\title{
Pensamiento indígena y medio ambiente en la jurisprudencia de las altas cortes de Colombia ${ }^{1}$
}

\section{Indigenous thought and the environment in the jurisprudence of the high courts of Colombia}

\begin{abstract}
Daniela López Madrid
Actualmente abogada. Cuando se escribió el artículo era estudiante de décimo semestre de Derecho en la Universidad Autónoma Latinoamericana, UNAULA, y perteneciente al semillero de investigación en Derecho Comparado. Email: danielalopezmadrid@gmail.com
\end{abstract}

Doi: https://doi.org/10.22267/rceilat.214849.97

\section{Resumen}

El constitucionalismo latinoamericano de los años 90, caracterizado por su manifestación utópica, permitió el reconocimiento de los derechos de los pueblos indígenas históricamente invisibilizados, además del reconocimiento de la ciudadanía y la garantía de la protección cultural, lingüística, jurídica y territorial.

Este último punto debe ser entendido más allá de una mirada política - jurisprudencial, ya que los resguardos indígenas incluyen, además de áreas delimitadas de vivienda, producción económica y extracción de recursos, una relación humana con el paisaje y el entorno. Por ello las Altas Cortes colombianas han explicado el vínculo entre las comunidades y la preservación de sus territorios desde la espiritualidad, la cosmovisión, la supervivencia y el aprovechamiento y protección del medio ambiente.

Palabras clave: Pensamiento indígena; Medio ambiente; Territorio; Altas cortes; Constitucionalismo.

\begin{abstract}
The Latin American constitutionalism of the 90s, characterized by its utopian manifestation, allowed the recognition of the rights of historically invisible indigenous people, in addition to the recognition of citizenship and the guarantee of cultural, linguistic, legal and territorial protection.
\end{abstract}

1 Ponencia derivada del proyecto de investigación titulado Construcción de Subjetividades Indígenas a partir del movimiento constitucional latinoamericano de los 90's, en actual desarrollo por el semillero de investigación en Derecho Comparado de la Facultad de Derecho de la Universidad Autónoma Latinoamericana, UNAULA, bajo la coordinación de la docente investigadora Diana Patricia Restrepo Ruiz. Ponencia presentada para la participación en la mesa temática La cuestión Étnica en Colombia, en el marco del XI Congreso Internacional de Pensamiento Latinoamericano: La Construcción de América Latina”. 
This last point must be understood beyond a political-jurisprudential view, since indigenous reservations include, in addition to delimited areas of housing, economic production and extraction of resources, a human relationship with the landscape and the environment. For this reason, the Colombian High Courts have explained the link between the communities and the preservation of their territories from the spirituality, worldview, survival and the use and protection of the environment.

Keywords: Indigenous thought; Environment; Territory; High courts; Constitutionalism

Colombia, según la Constitución Política de 1991, es un Estado fundado en el pluralismo y la interculturalidad. Estos principios fueron reconocidos formalmente a los Pueblos Indígenas por medio del Convenio 169 de la OIT $^{2}$ de 1989 firmado y aprobado por Colombia con la ley 21 de 1991, teniendo en cuenta los aspectos culturales y lingüísticos y su relación ancestral con el territorio que ocupan o apropian de alguna manera. Esta ratificación generó nuevas reformas constitucionales en los sistemas jurídicos de cada Estado parte, pues el mandato ordenaba reconocer jurídicamente el respeto por su identidad cultural y social, además de sus prácticas, costumbres, tradiciones e instituciones.

Así mismo, los artículos 13,14,15 y 16 de dicho Convenio, permitieron la

2 En junio de 1989, la Conferencia Internacional del Trabajo de la Oficina Internacional de Trabajo adoptó el Convenio núm. 169 sobre Pueblos indígenas y tribales en países independientes. A noviembre de 2014, el Convenio núm. 169 ha sido ratificado por 22 países, la mayoría de nuestra región: Argentina, Bolivia, Brasil, Chile, Colombia, Costa Rica, Dominica, Ecuador, Guatemala, Honduras, México, Nicaragua, Paraguay, Perú y la República Bolivariana de Venezuela, además de Dinamarca, España, Fiji, Nepal, Noruega, los Países Bajos y República Centroafricana. Tomado de https://www.ilo. org/wcmsp5/groups/public/---americas/---ro-li$\mathrm{ma} /$ documents/publication/wcms_345065.pdf consolidación de los derechos colectivos sobre las tierras y territorios ancestrales de los pueblos indígenas, donde será un deber de cada gobierno brindar protección efectiva del derecho de propiedad y posesión, y tomar las medidas que sean necesarias cuando persista alguna vulneración. Estos derechos comprenderían, además, la posibilidad de la utilización, administración y conservación de los recursos naturales en consideración de la relación cosmogónica y espiritual con la naturaleza.

Ahora bien, suele creerse que los problemas derivados de casi cinco siglos de desconocimiento, rechazo y aculturación de los pueblos indígenas fueron resueltos tras los reconocimientos internacionales y nacionales, pero a pesar de ser una manifestación pacífica y participativa, hemos visto aumentar problemas de difícil solución para los sistemas normativos, especialmente el colombiano.

Una de estas problemáticas es la concepción del ámbito territorial por parte de los órganos estatales, la cual ha sido superficial, espacial y jurisdiccional, pues el Estado en el ejercicio de sus funciones y según los mandatos internacionales, delimitó espacios los cuales serían ocupados por las comunidades indígenas, donde 
ejercerán su propia jurisdicción, normas, sanciones y demás, pero sin que estas sean contrarias a la Constitución y las leyes nacionales, por lo que "esta amenazante imposición de la norma sobre la realidad, muy posiblemente conducirá a los pueblos indígenas hacia posiciones erráticas, insostenibles para el colectivo, incluso a corto plazo”. (Hierro, 2004, pág. 278).

Lo anterior, ha generado que el Estado conciba a los indígenas desde el territorio y no desde el sujeto, creando así, nuevas subjetividades indígenas abigarradas, concepto zavaletiano que explica Antezana, (2009).

Se trata de la calificación mutua de diversidades económico-sociales de tal suerte que, en concurrencia, ninguna de ellas mantiene su forma (previa); la referencia, o sea, la sociedad concreta objeto de conocimiento permitiría caracterizar las diversas historias en juego, es decir, los diversos grados de constitución social (relativos) ahí implicados; y, el marco de "calificación de unas por otras" diversidades recurriría al concepto de "intersubjetividad" para reconocer, en las crisis sociales, el grado de unidad de la diversidad alcanzado en dicha concurrencia (p. 132).

Puede decirse entonces que, tras estos reconocimientos, en los sujetos indígenas concurrirán dos calidades: por un lado, serán sujetos occidentalizados, y, por otro lado, conservarán su propia identidad y cultura, pero, generando nuevos pensamientos $\mathrm{y}$ maneras de percibir su entorno; todo esto, por la implementación de los dispositivos de poder. ${ }^{3}$

Sin embargo, las comunidades indígenas optan por entender de diversas formas la territorialidad más allá del ámbito espacial. Varios autores han explicado la relación estrecha entre la naturaleza y el indígena, por ejemplo, Lévi- Strauss en su libro pensamiento salvaje, menciona, "la extremada familiarización con el medio biológico, la apasionada atención que le prestan, los conocimientos exactos a él vinculados, a menudo han impresionado a los investigadores (...)" (Lévi-Strauss, 1962). Esto es, los indígenas perciben el territorio como aquel espacio donde se construyen identidades e importantes vinculaciones espirituales con el medio que los rodea, creando así nuevas relaciones sociales y místicas que permiten la alta protección del medio ambiente y los recursos naturales que comprenden agua, montañas, animales, insectos, árboles, y también los espíritus del bosque.

A esta relación entre la naturaleza y la cultura indígena, se le llama animismo ${ }^{4}$, pues para los indígenas todos

3 Cf. Michel Foucault en su libro Tecnologías del Yo, explica cómo las tecnologías o dispositivos de poder por medio de la dominación estatal, terminan construyendo conductas o, en palabras suyas "objetivando el sujeto”. (Foucault, 1990, pág. 48)

4 Término que denota la creencia general de que todos los seres y objetos de la naturaleza están animados o tienen espíritu. Tomado de Word Reference. URL: https:// www.wordreference.com/definicion/animismo.

Explicado en términos del Antropólogo Philippe Descola, nuestra visión propia del mundo es dualista, ya que hace una 
lo que comprende el territorio está compuesto de alma al igual que ellos, pues perciben la naturaleza como un ser sintiente. Esto permite comprender que para los pueblos originarios "el espacio (...) es un nexo tangible con el pasado, algo que se puede ver, tocar y escalar, algo que funde en uno pasado y presente" (Rappaport, 2004, pág. 174), pues, cuando se reconocen espacios tangibles, más allá de la espiritualidad, se permite una organización espacial que conlleva a explicaciones históricas y cronológicas de resistencia y de lucha por parte de las comunidades indígenas en determinados territorios.

Estas resistencias y luchas van encaminadas justamente a la protección de sus hábitats naturales y territoriales, ya que el Estado, a pesar de haber titulado grandes extensiones de tierra a las comunidades indígenas y brindarles una mayor protección, también ha optado por la extracción y explotación de recursos naturales, además han sido despojados de sus tierras a causa del conflicto armado y han sido víctimas de etnocidios ${ }^{5} \mathrm{y}$ maltratos

distribución social y antropológica entre lo humano y lo no humano. Diferenciada del pensamiento indígena, basada en el animismo, pues su distribución está basada en la cosmología diferenciando hombres, plantas y animales, pero no lo hacen desde lo natural, si no desde un grado de diferenciación. Es más, para algunas culturas indígenas, la mayor parte de las plantas y animales están compuestos de alma. (Descola, 2004).

5 consistente en la desaparición forzada de una etnia por la destrucción de sus condiciones de vida y su sistema de creencias. (Sentencia T - 380, 1993) crueles e inhumanos, esto, solo por mencionar algunos factores de vulneración de los derechos de los pueblos indígenas, los cuales denotan la necesidad de acudir a la jurisdicción ordinaria con el fin de que se amparen dichos derechos.

A partir de ahí, es cuando vemos cómo los pueblos indígenas comienzan a hacer uso de los mecanismos de participación ciudadana, principalmente el de la consulta previa en materia medio ambiental, con el fin de proteger las sus territorios sagrados y ancestrales.

Así es como según la CEPAL "en bastantes casos todavía se duda sobre la necesidad de consultar a los pueblos indígenas" (CEPAL , 2014, pág. 26), aunque la Corte Constitucional en sentencia SU - $123 / 2018^{6}$, ha establecido la consulta previa como un derecho fundamental dotado del principio de inmediatez, derivado de la protección constitucional de la identidad étnica y cultural de los pueblos indígenas en materia medio ambiental.

Según lo anterior, las comunidades y pueblos indígenas se están manifestando por medios jurídicos como tutelas y demandas para exigir una protección real y jurídica de los terri-

6 "Este derecho implica que las comunidades indígenas y tribales deban ser consultadas sobre cualquier decisión que las afecte directamente, de manera que puedan manifestar su opinión sobre la forma y las razones en las que se cimienta o en las que se fundó una determinada medida, pues esta incide o incidirá claramente en sus vidas”. (Sentencia SU - 123, 2018) 
torios que habitan y que están siendo degradados por grandes industrias petroleras en búsqueda de recursos naturales con el fin de sacar provecho económico, ignorando la percepción ancestral del indígena con su hábitat. Del mismo modo, las comunidades manifiestan que existe una gran desigualdad jurídica en los derechos que se les han reconocido, puesto que aún existe ausencia de mecanismos realmente efectivos para obligar al Estado a cumplir con los preceptos internacionales de carácter vinculante. (Gómez-Rivera, 2014, pág. 150).

Es por esto que las Altas Cortes de Colombia en su jurisprudencia han venido explicando el vínculo y relación entre las comunidades y la preservación de sus territorios desde la espiritualidad, la cosmovisión, la supervivencia, el aprovechamiento y la protección del medio ambiente.

En este sentido, la sentencia arquimédica que mejor explica esta relación es la sentencia T - 622/2016 de la Corte Constitucional. El problema jurídico de esta sentencia, radicó en que las comunidades étnicas que se encuentran en territorios, los cuales, son ancestrales, se vieron directamente afectadas por la práctica de la minería y la deforestación, la cual estaba generando una alta contaminación en el Rio Atrato ${ }^{7}$ y como consecuencia

7 "El río Atrato es el más caudaloso de Colombia y también el tercero más navegable del país, después del río Magdalena y del río Cauca. El Atrato nace al occidente de la cordillera de los Andes, específicamente en el Cerro Plateado a 3.900 metros sobre graves enfermedades en las comunidades étnicas del lugar, especialmente en lo niños y niñas. Lo anterior, motivó a los representantes de dichas comunidades a instaurar acción de tutela, con el fin de que se ampararan sus derechos fundamentales a la vida, a la salud, al agua, a la seguridad alimentaria, al medio ambiente sano, a la cultura y al territorio de las comunidades étnicas accionantes.

La Corte Constitucional, de acuerdo con los antecedentes y las pretensiones de la parte demandante, decide ahondar sobre los Derechos Bioculturales, los cuales define como los derechos que tienen todas las comunidades étnicas de administrar los recursos naturales del territorio que habitan y en los cuales desarrollan su propia cultura de acuerdo a sus propias leyes, costumbres o tradiciones con base a la especial relación que tienen con el medio ambiente y la biodiversidad (Sentencia T - 622, 2016). Entonces, cuando hay prácticas que causen el deterioro o daño ambiental en los territorios ancestrales, se les arrebata a estas comunidades la oportunidad y la posibilidad de acceder a

el nivel del mar y desemboca en el golfo de Urabá, en el mar Caribe. Su extensión es de 750 kilómetros, de los cuales 500 son navegables. La parte más ancha del río tiene una longitud de 500 metros y en cuanto a la parte más profunda se estima cercana a los 40 metros. Recibe más de 15 ríos y 300 quebradas; entre los principales se cuentan: el Andágueda, Baté, Bojayá, Buchadó, Cabí, Cacarica, Capá, Domingodó, Napipí, Neguá, Muguindó, Murrí, Opogodó, Puné, Quito, Salaquí, Sucio, Tagachí y Truandó”. (Sentencia T - 622, 2016). 
los bienes individuales como la salud e integridad personal, vulnerando sus derechos bioculturales y su derecho al territorio, generando un desplazamiento de las comunidades étnicas a otros lugares del país.

Lo anterior lleva a La Corte a plantear la relevancia constitucional de la protección de los ríos, los bosques, las fuentes de alimento, el medio ambiente y la biodiversidad, pues tal como se planteó al inicio, nuestra Constitución está basada en principios como el pluralismo y la interculturalidad denotando el carácter de una Constitución Ecológica, que según la Sentencia C 431 del 2000 "está conformada por el conjunto de disposiciones superiores que fijan los presupuestos a partir de los cuales deben regularse las relaciones de la comunidad con la naturaleza y que propugnan por su conservación y protección".

Esta fue influenciada por instituciones internacionales con la finalidad que, las constituciones pertenecientes a organizaciones mundiales en pro de la protección del medio ambiente, protegieran el amplio rango de factores que componen la naturaleza y la biodiversidad como el agua, el aire, la tierra, la fauna, la flora, los ecosistemas, el suelo, el subsuelo, entre otros, ya que, estos factores nos han permitido la supervivencia humana, pues, la mayoría de bienes que utilizamos como suministro alimenticio provienen directamente de la naturaleza, y por parte de la biodiversidad recibimos beneficios como la regulación de los ciclos hídricos, del clima y servi- cios culturales. Esta visión es completamente biocéntrica ${ }^{8}$, pero contraria a la concepción antropocéntrica que estima que la naturaleza no es sujeto de derechos, sino un simple objeto de disposición del ser humano.

Entonces, la Corte Constitucional, basada en la concepción biocéntrica, considera finalmente,

Declarar la existencia de una grave vulneración de los derechos fundamentales a la vida, a la salud, al agua, a la seguridad alimentaria, al medio ambiente sano, a la cultura y al territorio de las comunidades étnicas que habitan la cuenca del río Atrato y sus afluentes, imputable a las entidades del Estado colombiano accionadas (Presidencia de la República, Ministerio de Interior, Ministerio de Ambiente y Desarrollo Sostenible, Ministerio de Minas y Energía, Ministerio de Defensa Nacional, Ministerio de Salud y Protección Social, Ministerio de Agricultura, Departamento para la Prosperidad Social, Departamento Nacional de Planeación, Agencia Nacional de Minería, Agencia Nacional de Licencias Ambientales, Instituto Nacional de Salud, Departamentos de Chocó y Antioquia, Corporación Autónoma Regional

8 Según Beatriz Ensabella "El biocentrismo va más allá de las posturas utilitaristas, donde todas las especies vivientes tienen la misma importancia. Es el reconocimiento del valor inherente de todas las formas de vida. De este modo, la Naturaleza es sujeto de valores, sujeto de derechos. Y en este sentido, se debe avanzar en la discusión de una ética ambiental en relación a una ética social que propenda a una justicia ambiental y ecológica”. (Ensabella, 2016 ) 
para el Desarrollo Sostenible del Chocó -Codechocó-, Corporación para el Desarrollo Sostenible del Urabá -Corpourabá-, Policía Nacional - Unidad contra la Minería Ilegal, y los municipios de Acandí, Bojayá, Lloró, Medio Atrato, Riosucio, Quibdó, Río Quito, Unguía, Carmen del Darién, Bagadó, Carmen de Atrato y Yuto -Chocó-, y Murindó, Vigía del Fuerte y Turbo -Antioquia-), por su conducta omisiva al no proveer una respuesta institucional idónea, articulada, coordinada y efectiva para enfrentar los múltiples problemas históricos, socioculturales, ambientales y humanitarios que aquejan a la región y que en los últimos años se han visto agravados por la realización de actividades intensivas de minería ilegal.

Y más importante aún, este alto tribunal considera "reconocer al río Atrato, su cuenca y afluentes como una entidad sujeto de derechos a la protección, conservación, mantenimiento y restauración a cargo del Estado y las comunidades étnicas, conforme a lo señalado en la parte motiva de este proveído en los fundamentos". (Sentencia T - 622, 2016).

Por otro lado, el pueblo indígena Barí, perteneciente a la familia lingüística Arawak y que habita en el departamento Norte de Santander, representado por la Asociación de Autoridades Tradicionales del Pueblo Barí NATUBAIYIBARI, decide instaurar acción de tutela contra el INCODER, en razón de que desde el año 2005 el pueblo indígena Barí ha promovido y realizado diversas acciones tendientes a la recuperación de los territorios an- cestrales, que han perdido a causa de la presencia de cultivos ilícitos y de la guerra entre actores armados, y demás hechos causantes de desplazamiento que han generado la disminución del volumen poblacional.

Sin embargo, la Procuraduría Delegada para Asuntos Ambientales y Agrarios decidió responder de manera positiva a las acciones instauradas, ya que, para esta entidad, el derecho al territorio de los pueblos indígenas se hace extensivo a todas las tierras que hayan poseído en el pasado y que estén haciendo uso en el presente, sin importar si fueron despojados o no, puesto que el despojo no es una causa para romper la memoria colectiva y ancestral con el territorio. Según esto, los Estados y entidades encargados están obligados a otorgar tierras con extensión suficiente para la conservación y desarrollo de sus formas de vida; además, el derecho a ser reconocidos como dueños debe hacerse a través de un título jurídico actual y plenamente válido.

Finalmente, invocaron en favor del pueblo Barí las medidas de protección contenidas en el auto A-004 de 2009 de esta corporación, en el que, como parte del proceso de seguimiento a la protección otorgada a familias desplazadas, la Corte adoptó medidas especiales para enfrentar, desde un enfoque diferencial, las situaciones de desplazamiento y problemas relacionados, que afectan, de manera incluso más intensa, a los miembros de los pueblos indígenas. 
En esta sentencia, también se observa una postura biocéntrica por parte de la Corte Constitucional, puesto que, en lo ateniente del derecho al territorio y a la propiedad colectiva de la tierra, menciona el sólido vinculo de pertenencia de los pueblos indígenas con el territorio pues, para la Corte, el sentido de comunidad de las comunidades indígenas es más fuerte por factores como la espiritualidad y la cosmovisión por la autosuficiencia alimentaria del aprovechamiento del territorio, más que en otras comunidades. "Por ello, además de lo atinente a la titulación de las tierras, punto que en este caso no resulta relevante, es también de la mayor importancia para estas comunidades el pleno y tranquilo disfrute de sus territorios, sin lugar a ninguna clase de interferencias externas”. (Sentencia T - 052, 2017).

Otro aspecto relevante de esta sentencia que se ha planteado es la intervención de la ONIC ${ }^{9}$. Esta organización expresó su opinión sobre esta acción de tutela, respaldando las pretensiones planteadas por la parte demandante realizando una reflexión sobre los derechos fundamentales invocados en base al Convenio 169 de la OIT, principalmente refiriéndose sobre el derecho al territorio, el derecho a la consulta previa, el debido proceso y el derecho a la integridad étnica y cultura de la comunidad indígena.

9 Organización Nacional Indígena de Colombia. En la actualidad, son filiales 47 organizaciones zonales y regionales, que se encuentran en 28 de los 32 departamentos del país. Tomado de la página oficial de la ONIC URL: https://www.onic.org.co/onic
Igualmente, en la Sentencia T 849 del 2014, la comunidad perteneciente al Resguardo Indígena Arhuaco de la Sierra Nevada de Santa Marta, instauró acción de tutela contra el Ministerio del Interior, la Corporación Autónoma Regional del Cesar, Agregados del Cesar EU, y Pavimentos y Construcciones El Dorado LTDA Ingenieros Contratistas, manifestando que, a pesar de la delimitación geográfica que realizó el Ministerio del Interior, la cual llamó línea negra ${ }^{10}$, se otorgó licencia por parte de la Corporación Autónoma Regional del Cesar para la explotación de un yacimiento minero, esto, sin haberse practicado el procedimiento de la consulta previa, ya antes mencionada.

En este caso, puede denotarse una gran vulneración de derechos fundamentales de las comunidades indígenas, especialmente, el de la consulta previa y el derecho de propiedad, siendo éste de carácter colectivo y que tiene una relación directa con el desarrollo cultural, la vida en comunidad y la proyección social de las mismas. Aunque algunas entidades lograron demostrar que el lugar donde se estaba realizando la explotación no estaba

10 Para la comunidad arahuaca, la Línea Negra (sei-shizha), constituye el Territorio Tradicional y sagrado para las comunidades indígenas existentes en la Sierra Nevada de Santa Marta. Esta línea demarca los lugares de vital importancia para hacer los pagamentos y ritos ceremoniales, que tienen importancia fundamental en el equilibrio ecológico y ambiental de la naturaleza, para de esta manera evitar sequías, terremotos, inundaciones, enfermedades etc. (Sentencia T - 634 , 1999) 
habitado por indígenas, la Corte Constitucional decide explicar el concepto de territorio indígena y su protección especial cuando se trata de áreas sagradas y de importancia cultural para las comunidades, incluso cuando se trata de zonas fuera de los resguardos titularizados, para esto cita la Sentencia T - 693 del 2011, la cual expresa la amplia protección de áreas sagradas sin importar si se encuentran fuera de los resguardos, puesto que según el Convenio 169 "acoge un concepto amplio de territorio, al indicar que se consideran como tal, aquellas áreas de una comunidad que comprenden, no sólo las tituladas o habitadas, sino también aquellas que constituyen el ámbito tradicional de sus actividades tradicionales, sagradas o espirituales" (Sentencia T - 693, 2011).

Esto es, la protección ambiental que debe ejercer el Estado y sus entidades delegadas, no solo se aplicará a los territorios indígenas previamente delimitados, sino también, a aquellos territorios los que, por alguna razón, estén relacionados con sus costumbres y rituales.

Otro aspecto importante de la sentencia que resuelve esta problemática es la intervención de Danilo Villafañe indígena Arhuaco, el cual construye el concepto de territorio, a partir de sus vivencias en la Sierra Nevada de Santa Marta. De manera organizada, explica que el concepto de territorio debe incorporar siete categorías basadas en la pertenencia ancestral y el derecho a la posesión, que pueden expresarse de manera literal, así:
"1. Su Historia, basada en la continuidad de la realización de las funciones asignadas, y en la que el pasado ordena, por tradición, el equilibrio necesario. En este sentido, una visión progresista que deslegitime o menosprecie la importancia de ese pasado no tiene cabida en su pensamiento cultural. El Sé es la ley tradicional para el orden territorial decidido en los zwama.

2. La interdependencia entre el territorio y la cultura tradicional indígena.

3. Las prácticas sagradas, basadas en la Ley ancestral que garantizan el mantenimiento del equilibrio.

4. La vida, tanto del territorio como de todo lo que él contiene por la Ley del sé.

5. El orden, dado por la compleja estructura articulada por los sitios, conocimientos y prácticas determinadas por la Ley tradicional.

6. La responsabilidad y competencia ejercida al interior y hacia el exterior por las autoridades indígenas - mamos, makú y mayores-.

7. Lo ancestral, pues el territorio y su conocimiento están inmersos en la cultura de los cuatro pueblos de la Sierra Nevada desde los orígenes míticos.”. (Sentencia T - 849, 2014). Esta concepción de territorio, viene siendo la más estructurada; del mismo modo, en la Sentencia T - 634 de 1999, Según la Comunidad Arahuaca ancestral:

\footnotetext{
"Cuando los indígenas hablamos del territorio tradicional, hacemos referencia al espacio donde se recrea la cultura, a la integridad de relaciones sociales, culturales y espirituales que constituyen la base
} 
de nuestra permanencia como etnia. Razón por la cual las luchas indígenas se han enfocado a recuperar el territorio y tener un control, fundamentado en la ocupación ancestral de los espacios, porque nos representa la garantía para seguir existiendo como pueblos, porque de aquí depende la vida, cultura y toda clase de conocimiento".

El anterior rastreo jurisprudencial, nos permite comprender la forma cómo el pensamiento indígena ha sido reivindicado por la Corte Constitucional en materia medioambiental, pues este órgano ha explicado, en muchos de sus pronunciamientos, esta relación no solo a partir de los preceptos internacionales y leyes nacionales (que claro, son incluyentes y permiten una consolidación formal), sino a partir de las subjetividades y vivencias indígenas, tal como se hace en las sentencias T - 634 de 1999 y la $\mathrm{T}-849$ del 2014.

Además, se demuestra lo expuesto en el boletín número 3 del Grupo de Trabajo de Naciones Unidas sobre Derechos sobre Recursos Tradicionales de 1996: las demandas que más se presentan por parte de las comunidades indígenas son referentes a la autodeterminación y la protección de sus territorios (Aguilar, 2003, pág. 62), que si bien, estas demandas se expresan a través del mecanismo de la acción de tutela y que, la mayoría han sido resueltas a favor de los pueblos indígenas, sin embargo, se cuestiona la idea de cuán eficaz han sido todos estos pronunciamientos jurisprudenciales que si bien, protegen los dere- chos de los pueblos indígenas y explican la relación con el entorno, vemos cómo aumentan las vulneraciones hacia estas comunidades por razones como: las entidades estatales aun sostienen el término occidental de territorio basado en la propiedad privada, y que se contrapone notablemente con el pensamiento indígena, puesto que para ellos no existe la propiedad privada porque no se apropian de las cosas materiales (Hierro, 2004), esto genera una discrepancia, ya que, el juez constitucional está resolviendo los asuntos a favor de las comunidades indígenas desde una interpretación biocéntrica otorgándoles un sin número de derechos y reconociendo sus territorios ancestrales, declarando y ordenando a las entidades accionadas o demandadas, dependiendo del caso en concreto, a cesar el daño o repararlo de alguna forma. Sin embargo, estas entidades han hecho caso omiso a los títulos ejecutivos, persistiendo la vulneración basada en intereses económicos y políticos.

Es allí entonces donde se pone en duda el concepto de interculturalidad planteado inicialmente. Juan Álvaro Echeverri lo plantea como un concepto aparente donde puede existir una visión territorial y no ambiental. Lo ideal sería entonces, construir un concepto de interculturalidad que no solo implique el reconocimiento y el valor, si no el hecho de adoptarlo como propio, experimentarlo o re- crearlo (Echeverri, 2004, pág. 266).

Finalmente, el constitucionalismo de los años 90's es el que ha permi- 
tido muchos reconocimientos y nuevas miradas utópicas acerca de los pueblos indígenas, sin embargo, este constitucionalismo sigue permeado de una visión occidental, que genera diferencias con el pensamiento indígena y el respeto por la naturaleza y el medio que los rodea; aunque este idioma occidental plantea un lenguaje de dignidad, tal como lo plantea Boaventura de Sousa Santo en su ponencia en la VII Conferencia Latinoamericana y Caribeña de Ciencias Sociales "Transformaciones democráticas, justicia social y procesos de paz", del 9 al 13 de noviembre de 2015 en Medellín,
Colombia $^{11}$, NO SON COMPLETAMENTE UNIVERSALES, ya que este idioma o percepción no comprende la espiritualidad de los pueblos indígenas, y allí es cuando persiste hoy en día la vulneración del derecho al territorio, porque aún se implantan ideas hegemónicas en las sentencias de las Altas Cortes de Colombia, que siguen estando muy alejadas de su propia realidad y cosmovisión.

11 Tomado de YouTube. Boaventura de Sousa Santos - Un diálogo sobre los derechos humanos y la paz

URL: https://www.youtube.com/watch?$\mathrm{v}=$ pxbrkStZmis\&feature $=$ youtu.be

\section{Bibliografía}

Aguilar, G. (Octubre de 2003). Proyecto Regional de Manejo Integrado de Ecosistemas por Pueblo Indígenas y Comunidades de Centroamérica. Conocimiento Tradicional sobre la Biodiversidad en el Proyecto Manejo Integrado de Ecosistemas por Pueblo Indígenas y Comunidades. GEF-PPG-No. 051362.

Antezana, L. H. (Septiembre de 2009). Biblioteca CLACSO. Obtenido de http://biblioteca. clacso.edu.ar/clacso/coediciones/20160315033915/07antezana.pdf

CEPAL . (2014). Conferencia Mundial de los Pueblos Indígenas. Los Pueblos Indígenas en América Latina (pág. 26). Santiago de Chile: Limitada .

Descola, P. (2004). Las cosmologías indígenas de la Amazonía . En A. S. Hierro, TIERRA ADENTRO: territorio indígena y percepción del entorno (págs. 25-35). Copenhague: IWGIA .

Echeverri, J. Á. (2004). Territorio como cuerpo y territorio como naturaleza: ¿Diálogo intercultural? En A. S. Henao, Tierra adentro: territorio indígena y percepción del entorno (págs. 259 - 275). Copenhage : IWGIA .

Ensabella, B. (2016 ). Derechos de la naturaleza. Ética biocéntrica y políticas ambientales . Revista Latinoamericana , 638-688.

Foucault, M. (1990). Tecnologías del yo y otros textos afines . Barcelona : Paidós Ibérica S.A. .

Gómez-Rivera, M. (Julio de 2014). Red de biblioteca CLACSO. Obtenido de http://biblioteca.clacso.edu.ar/Mexico/cieap-uaem/20170424052329/pdf 495.pdf

Hierro, P. G. (2004). Territorios Indígenas: tocando a las puertas del derecho . En A. S. Hierro, TIERRA ADENTRO: territorio indígena y percepción del entorno (pág. 278). Copenhague: IWGIA .

Lévi-Strauss, C. (1962). PENSAMIENTO SALVAJE . París: FONDO DE CULTURA ECONÓMICA. 
Rappaport, J. (2004). La geografía y la concepción de la historia de los Nasa. En A. S. Hierro, TIERRA ADENTRO territorio indígena y percepción del entorno (pág. 174). Copenhague: IWGIA.

Sentencia C - 431 (Corte Constitucional 2000).

Sentencia SU - 123 (Corte Constitucional 2018).

Sentencia T - 052 (Corte Constitucional 2017).

Sentencia T - 380 (Corte Constitucional 1993).

Sentencia T - 622 (Corte Constitucional 2016).

Sentencia T - 634 (Corte Constitucional 1999).

Sentencia T - 693 (Corte Constitucional 2011).

Sentencia T - 849 (Corte Constitucional 2014). 